\title{
Bacterial meningitis and multiple abscess formation in the iliopsoas, erector spinae, and vastus lateralis muscle in a maintenance hemodialysis patient treated with continuous epidural anesthesia for herpes zoster-related pain control: a case report and review of the literature
}

Shunsuke Yamada ${ }^{1}$, Narihito Tatsumoto ${ }^{1}$, Noriko Nakamura' ${ }^{1},{ }^{\prime}$ Kosuke Masutani ${ }^{1}$, Toshiro Maeda ${ }^{2}$, Takanari Kitazono ${ }^{1}$ and Kazuhiko Tsuruya ${ }^{1,3^{*}}$

\begin{abstract}
Background: Infection is the second leading cause of mortality in patients who undergo maintenance hemodialysis. In this population, impairment in both cellular and humoral immunity contributes to the increased incidence of infection and infection-related hospitalization. However, these artificial devices occasionally enhance the risk of deep organ infection including muscle abscess, frequently leading to disability and mortality in hemodialysis patients.

Case presentation: A 54-year-old male undergoing maintenance hemodialysis was hospitalized because of the acute onset pain in the back and bilateral legs and declining consciousness which started at 7 days after the treatment of herpes zoster-related neuralgia with continuous epidural anesthesia. Physical examination revealed purulent discharge from the insertion site of the catheter. Serum biochemical tests showed increased inflammatory response and malnutrition. Magnetic resonance imaging revealed meningitis and multiple abscesses in the iliopsoas, erector spinae, gluteus medius, and vastus lateralis muscles, where conventional antibiotic treatment often fails to cure. Staphylococcus aureus was detected in the cerebrospinal fluid. Combination of intravenous antibiotics treatment and aggressive open surgical drainage of the muscle abscesses finally cured meningitis and multiple deep muscle abscesses in this patient.

Conclusions: Treatment with sensitive antibiotics and timely open surgical drainage is necessary to cure catheterrelated meningitis and secondary deep muscle abscesses in hemodialysis patients. Our case also suggests that epidural anesthesia may not be a good therapeutic option for herpes zoster because patients with herpes zoster are immunologically more compromised during and shortly after reactivation of varicella zoster virus.
\end{abstract}

Keywords: Abscess, Epidural catheter, Erector spinae muscle, Hemodialysis, lliopsoas muscle, Vastus lateralis muscle

\footnotetext{
* Correspondence: tsuruya@intmed2.med.kyushu-u.ac.jp

'Department of Medicine and Clinical Science, Graduate School of Medical Sciences, Kyushu University, 3-1-1 Maidashi, Higashi-ku, Fukuoka 812-8582, Japan

${ }^{3}$ Department of Integrated Therapy for Chronic Kidney Disease, Graduate School of Medical Sciences, Kyushu University, 3-1-1 Maidashi, Higashi-ku, Fukuoka 812-8582, Japan

Full list of author information is available at the end of the article
}

(c) The Author(s). 2018 Open Access This article is distributed under the terms of the Creative Commons Attribution 4.0 International License (http://creativecommons.org/licenses/by/4.0/), which permits unrestricted use, distribution, and reproduction in any medium, provided you give appropriate credit to the original author(s) and the source, provide a link to the Creative Commons license, and indicate if changes were made. The Creative Commons Public Domain Dedication waiver (http://creativecommons.org/publicdomain/zero/1.0/) applies to the data made available in this article, unless otherwise stated. 


\section{Background}

Infection is the second leading cause of mortality, accounting for $18-25 \%$ of deaths in patients who undergo hemodialysis $[1,2]$. Impairment in both cellular and humoral immunity contributes to the increased incidence of infection and infection-related hospitalization in hemodialysis patients [3-5]. Besides, dialysis patients have greater opportunities to receive various types of artificial medical devices for treatment and examination, catheterization for angiography and intravascular placement of catheters, and artificial grafts for dialysis access. These artificial devices greatly enhance the risk of deep organ infection including muscle abscess, frequently leading to disability and mortality in hemodialysis patients [6-10].

Herpes zoster is a self-limited dermatomal rash related to reactivation of the varicella zoster virus (VZV) in the dorsal root ganglion [11]. A recent population-based cohort study showed that hemodialysis patients are at 2.3 times higher risk of herpes zoster compared with the general population [12]. Standard treatment for herpes zoster and its related neuralgia includes anti-viral drugs (aciclovir, valaciclovir, famciclovir) and analgesics such as narcotics, topical lidocaine treatment, anticonvulsants, and nerve blocking [13-15]. Since anti-viral drugs are metabolized and secreted via the kidney, the dose should be reduced in dialysis patients. When patients with herpes zoster suffer from severe pain, epidural anesthesia is occasionally applied. However, when patients are suffering from active herpes zoster, they are immunologically more compromised than when they are not affected by that disease and put at an increased risk of infection. Here, we have a clinically important question whether epidural anesthesia is a safe treatment option for the pain control in hemodialysis patients with active herpes zoster.

We herein report the case of a patient undergoing maintenance hemodialysis who developed acute bacterial meningitis and multiple deep muscle abscesses related to epidural anesthesia during pain control for herpes zoster.

\section{Case presentation}

A 54-year-old male was hospitalized for the diagnosis and treatment for fever, backache, bilateral leg pain, and declining consciousness. He had been on maintenance hemodialysis due to endstage kidney disease with unknown etiology since he was 29 years old. At ages 46 and 49 , he received surgeries for carpal tunnel syndrome secondary to dialysis-related amyloidosis. He received also total parathyroidectomy with auto-transplantation in the right forearm for the treatment of secondary hyperparathyroidism at age 47 . He neither had a history of diabetes mellitus and immune deficiency nor received immunosuppressants. He had been on maintenance dialysis with the use of his arteriovenous fistula as a vascular access.

Three weeks prior to the admission, he developed herpes zoster in the left inguinal and groin area (L1-L2) and in the left waist area (L1-L2) and was treated with reduced dose of valacyclovir ( $250 \mathrm{mg} /$ day $)$. Because herpes zoster-related neuralgia was so severe and nonsteroidal anti-inflammatory drugs were ineffective, he requested a more effective pain control and was then treated with continuous epidural anesthesia for the relief of severe pain. Seven days after starting continuous epidural anesthesia, epidural catheter was removed. At the time of catheter removal, purulent discharge was observed at the insertion site of epidural catheter. Although levofloxacin was initiated thereafter, he rapidly developed backache and bilateral leg pain and declining consciousness on the next day of catheter removal and was transferred to our hospital.

On admission, consciousness level was II-10 in the Japan Coma Scale. His height was $170 \mathrm{~cm}$, his body weight was $62 \mathrm{~kg}$, and his body mass index was $21.5 \mathrm{~kg} / \mathrm{m}^{2}$. Blood pressure was $156 / 84 \mathrm{mmHg}$ in supine position. Heart rate was $104 \mathrm{bpm}$. Body temperature was $38.4{ }^{\circ} \mathrm{C}$. A summary of blood test is shown in Table 1 including blood hemoglobin level of $106 \mathrm{~g} / \mathrm{L}$, white blood cell count of $28,600 / \mu \mathrm{L}$ (neutrophil $89.5 \%$ ), platelet count of $8.7 \times$ $10 \% / \mu \mathrm{L}$, serum albumin level of $29 \mathrm{~g} / \mathrm{L}$, and C-reactive protein level of $215 \mathrm{nmol} / \mathrm{L}$. These results suggested mild level of anemia and malnutrition, elevated inflammatory response, and the presence of bacterial infection.

Plain computed tomography did not show any mass lesion in the head. Because bacterial meningitis was highly suspected, cerebrospinal fluid was collected by lumbar puncture. The findings of the cerebrospinal fluid showed increased cell count with higher neutrophil dominance, increased levels of protein and lactate dehydrogenase, and decreased levels of glucose, indicating bacterial infection (Table 2).

Magnetic resonance imaging was performed for further evaluation. In the T2-weighted imaging, round high-intensity lesion at the L4/L5 level, indicative of epidural abscess, was observed (Fig. 1a). Also, round high-intensity lesions in the left iliopsoas muscle and in the bilateral erector spinae muscles were detected (Fig. 1b). Finally, he was diagnosed with bacterial meningitis and multiple abscesses in the iliopsoas and erector spinae muscles, which were probably triggered by the indwelling catheter for continuous epidural anesthesia.

Treatment with immunoglobulin (5 g/day for 3 days), antibiotics (vancomycin, 2 g/week; ampicillin 1 g/day; ceftriaxone, $2 \mathrm{~g} /$ day), and phenytoin were initiated on the first hospital day. On the fifth hospital day, blood 
Table 1 Laboratory data on admission

\begin{tabular}{|c|c|c|c|c|c|c|c|c|}
\hline Complete blood count & Value & Unit & Serum biochemistries (continued) & Value & Unit & Immunology & Value & Unit \\
\hline Hemoglobin & 106 & $g / L$ & Sodium & 139 & $\mathrm{mmol} / \mathrm{L}$ & HBs antigen & - & \\
\hline Hematocrit & 32.8 & $\%$ & Potassium & 5.8 & $\mathrm{mmol} / \mathrm{L}$ & HBs antibody & - & \\
\hline White blood cell & 28.6 & $10^{9} / \mathrm{L}$ & Chloride & 99 & $\mathrm{mmol} / \mathrm{L}$ & HCV antibody & + & \\
\hline Neutrophils & 89.5 & $\%$ & Lactate dehydrogenase & 412 & $U / L$ & HTLV-1 antibody & + & \\
\hline Platelet count & 87 & $10^{9} / \mathrm{L}$ & Amylase & 246 & $U / L$ & TPHA test & - & \\
\hline Serum biochemistries & & & Creatinine kinase & 443 & $U / L$ & Coagulation & & \\
\hline Total protein & 62 & $g / L$ & Calcium & 2.33 & $\mathrm{mmol} / \mathrm{L}$ & Fibrinogen & 15.6 & $\mu \mathrm{mol} / \mathrm{L}$ \\
\hline Albumin & 29 & $g / L$ & Phosphate & 1.94 & $\mathrm{mmol} / \mathrm{L}$ & FDP & 16.5 & $\mathrm{mg} / \mathrm{L}$ \\
\hline Blood urea nitrogen & 39.3 & $\mathrm{mmol} / \mathrm{L}$ & Magnesium & 1.2 & $\mathrm{mmol} / \mathrm{L}$ & D-dimer & 4.9 & $\mu \mathrm{g} / \mathrm{mL}$ \\
\hline Creatinine & 875 & $\mu \mathrm{mol} / \mathrm{L}$ & Iron & 3.0 & $\mu \mathrm{mol} / \mathrm{L}$ & PT-INR & 1.34 & \\
\hline Uric acid & 595 & $\mu \mathrm{mol} / \mathrm{L}$ & Total cholesterol & 2.87 & $\mathrm{mmol} / \mathrm{L}$ & APTT & 36.7 & s \\
\hline Total bilirubin & 5.1 & $\mu \mathrm{mol} / \mathrm{L}$ & Triglyceride & 1.79 & $\mathrm{mmol} / \mathrm{L}$ & APTT-control & 35.7 & s \\
\hline Direct bilirubin & 1.7 & $\mu \mathrm{mol} / \mathrm{L}$ & HDL-cholesterol & 0.36 & $\mathrm{mmol} / \mathrm{L}$ & Blood gas analysis & & \\
\hline Alanine aminotransferase & 27 & $U / L$ & Glucose & 4.2 & $\mathrm{mmol} / \mathrm{L}$ & $\mathrm{pH}$ & 7.42 & \\
\hline Aspartate aminotransferase & 14 & U/L & C-reactive protein & 215 & $\mathrm{nmol} / \mathrm{L}$ & $\mathrm{pO}_{2}$ & 73.3 & $\mathrm{mmHg}$ \\
\hline Alkaline phosphatase & 586 & $U / L$ & & & & $\mathrm{pCO}_{2}$ & 45.1 & $\mathrm{mmHg}$ \\
\hline Gamma-glutamyl transferase & 186 & $U / L$ & & & & $\mathrm{HCO}_{3}^{-}$ & 28.4 & $\mathrm{mmol} / \mathrm{L}$ \\
\hline
\end{tabular}

$A P T T$ activated partial thrombin time, $H B$ s hepatitis B surface, FDP fibrin/fibrinogen degradation product, $H C V$ hepatitis $C$ virus, $H D L$ high-density lipoprotein, HTLV-1 human T cell leukemia/lymphoma virus-1, PT-INR internationalized prothorombin time, TPHA Treponema pallidum hemagglutinin

culture turned out to be negative. However, culture result of cerebrospinal fluid disclosed Staphylococcus aureus. The Staphylococcus aureus was sensitive to cefazolin, gentamycin, amikacin, erythromycin, clindamycin, minocycline, vancomycin, teikoplanin, levofloxacin, and sulfamethoxazole/trimethoprim but resistant to ampicillin. Accordingly, ampicillin was discontinued and clindamycin was added instead. However, high fever lasted and serum inflammatory markers did not decrease. Hence, minocycline and sulfamethoxazole were added on after consulting our infection team. On the 14th hospital day, cell count and lactated dehydrogenase level in the cerebrospinal fluid decreased compared to the initial evaluation. His consciousness returned to almost normal.

Table 2 Findings of cerebrospinal fluid on admission

\begin{tabular}{ll}
\hline & Values \\
\hline Cell count & $14,330 / 3$ \\
Monocyte, \% & 20 \\
Segmented, \% & 80 \\
Total protein, mg/dL & 345 \\
Lactate dehydrogenase, U/L & 788 \\
Glucose, mg/dL & 2 \\
Sodium, mmol/L & 140 \\
Potassium, mmol/L & 4.9 \\
Chloride, mmol/L & 108 \\
\hline
\end{tabular}

Analysis of the cerebrospinal fluid obtained on the first hospital day is shown
However, fever lasted and serum levels of C-reactive protein remained high. At this point, echocardiography did not show any evidence of infective endocarditis, but gallium scintigraphy showed a strong accumulation around the left hip joint. Magnetic resonance imaging performed on the 19th hospital day showed bursitis around the femoral head and pyomyositis in the left gluteus minimus and medius muscles, large abscess in the left vastus lateralis muscle, which was probably spread from the abscess in the left iliopsoas muscle (Fig. 2). Although intravenous antibiotic administration (meropenem $1 \mathrm{~g} /$ day and vancomycin $2 \mathrm{~g} /$ week) was continued, multiple abscesses did not shrink. In addition, repeated culture of blood and cerebrospinal fluid did not detect any bacteria. Accordingly, surgical open drainage was performed on the 48th hospital day, followed by a drastic shrink of abscess in the vastus lateralis muscle and a decrease in serum inflammatory markers. Meropenem (1 g/day) was continued even after abscess disappeared. During the hospitalization, he also developed disuse atrophy and needed rehabilitation because of the long-term bedridden condition. On the 108th hospital day, he was finally transferred to another hospital for rehabilitation and maintenance hemodialysis. At 1 year after his discharge, he had completely recovered from multiple deep muscle abscesses and was on maintenance hemodialysis without relapse. Clinical course during the hospitalization is summarized in Fig. 3. 

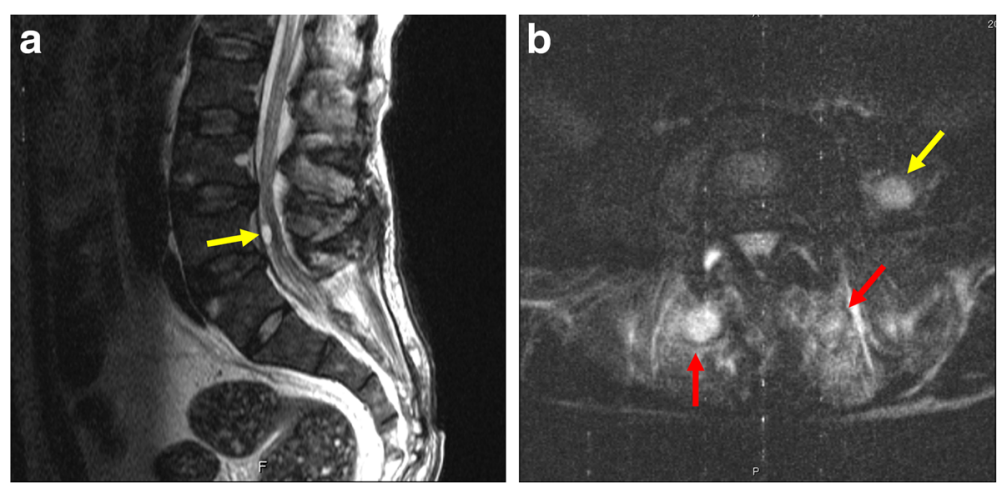

Fig. 1 Magnetic resonance imaging of the sagittal and cross-sectional spine. a Epidural abscess (yellow arrow) shown as a rounded high-intensity lesion at the L4/L5 level. b Abscess in the left iliopsoas muscle (yellow arrow, round high-intensity lesion) and bilateral erector spinae muscles (red arrows, round high-intensity lesions). L lumbar

\section{Discussion}

Herpes zoster is a process of reactivation of VZV which remains dormant in the sensory ganglia of the cranial nerve or the dorsal root ganglia after a previous varicella infection $[16,17]$. Epidural anesthesia, occasionally used for pain control caused by various disorders in the general population, is also effective in hemodialysis patients. However, when patients are being affected by herpes zoster, it is unclear whether epidural anesthesia for pain control is a safe treatment, especially in hemodialysis patients. Generally, patients with active herpes zoster are immunologically being more compromised than when they are not affected by reactivation of VZV. As for hemodialysis patients, they are immunologically compromised because of their impaired immunity by uremic milieu. Besides, artificial devices such as catheter increase the risk of infection. Considering these medical conditions, epidural anesthesia may not be a good therapeutic option for the control of herpes zoster-related pain in hemodialysis patients with active herpes zoster, potentially causing severe infection as in the present case. Hence, further studies are necessary to determine
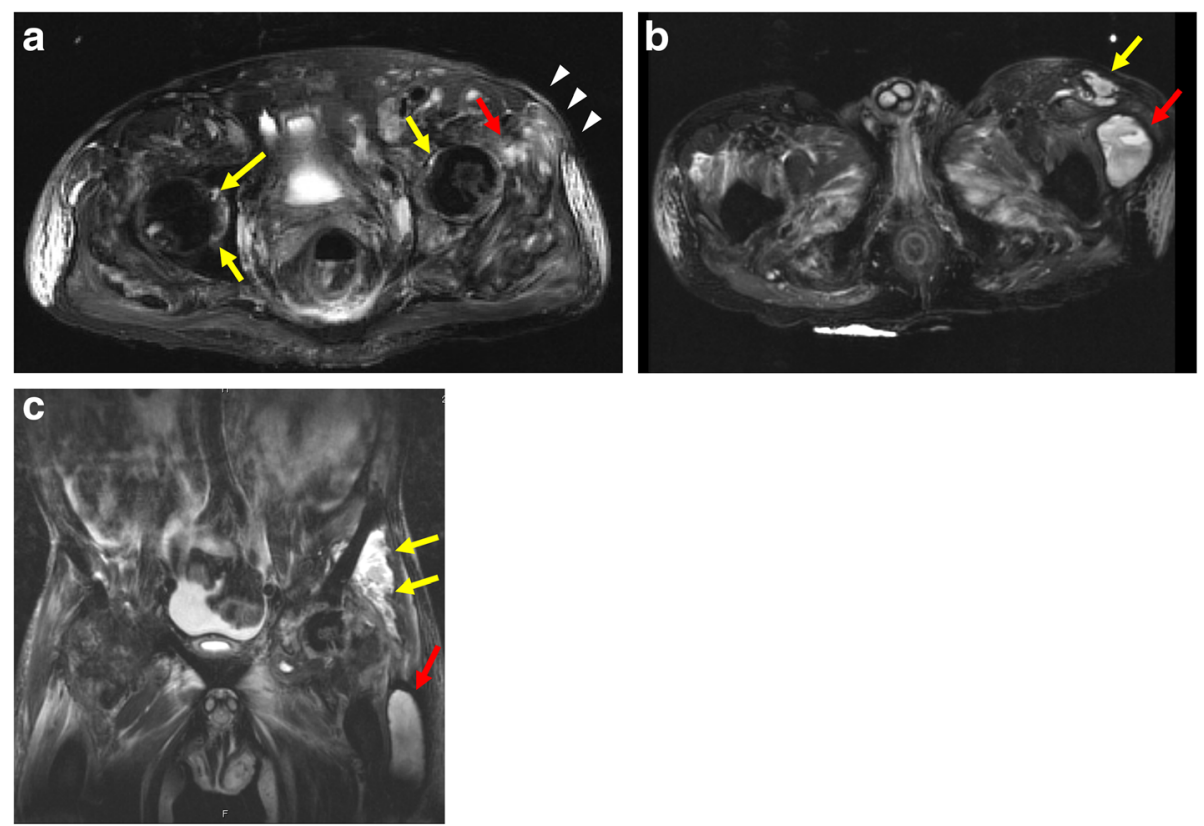

Fig. 2 Magnetic resonance imaging of the abdomen. a Bursitis around hip joints (yellow arrows) and pyomyositis in the left gluteus minimus (red arrow) and gluteus medius muscle (white arrow heads). b Abscess in the left gluteus medius (yellow arrow) and vastus lateralis muscle (red arrow) (cross-section). c Abscess in the left gluteus minimus and medius muscles (yellow arrow) and vastus lateralis muscle (red arrow) (coronal section) 


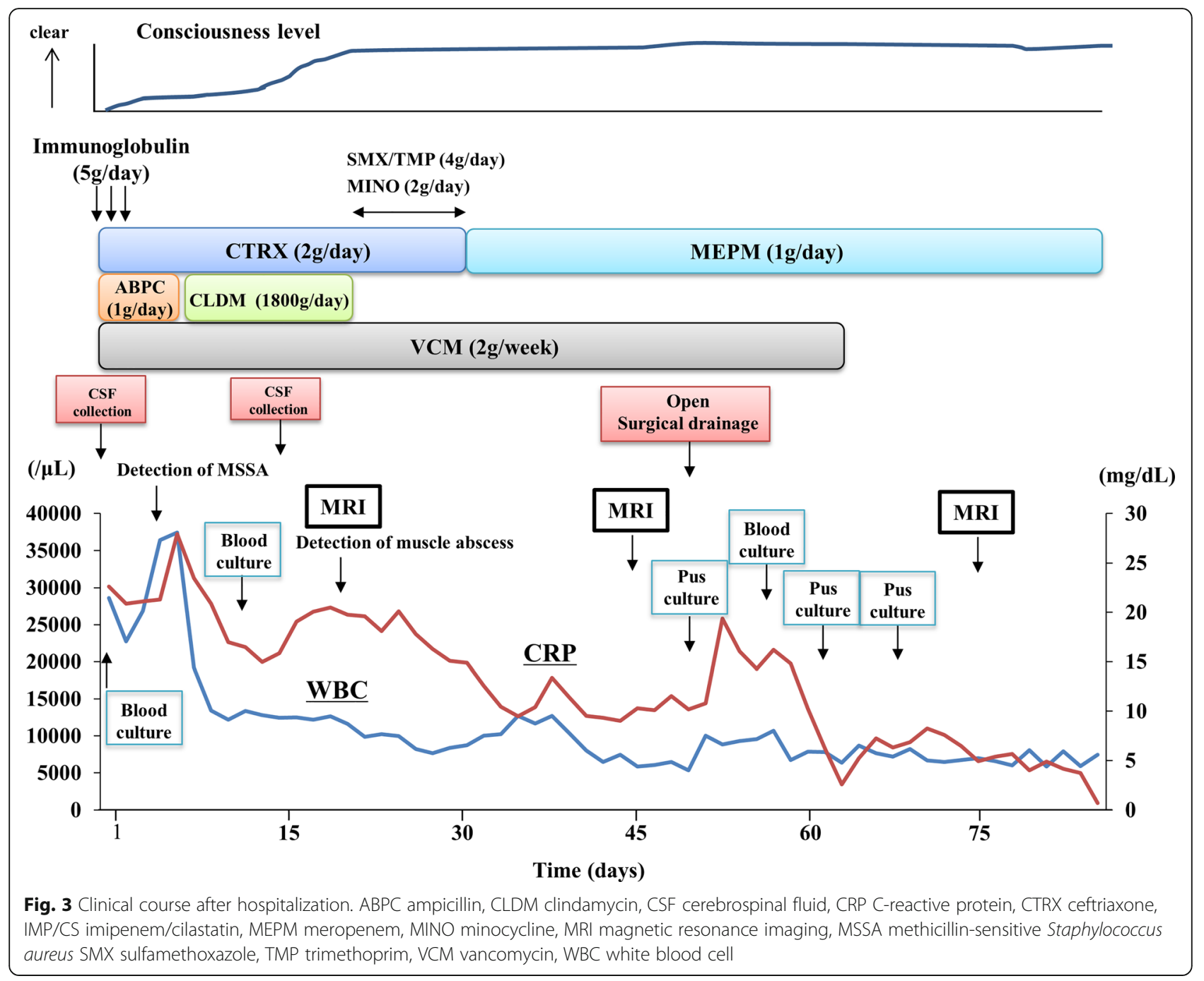

whether the risk for epidural catheter-related infection in hemodialysis patients with active herpes zoster is higher than those without active herpes zoster.

Psoas muscle abscess is a rare but lethal complication that is occasionally seen in hemodialysis patients [8-10, 18-28]. Triad of the classical psoas muscle abscess consists of fever, pain, and limp [29]. Psoas muscle abscess is generally classified into primary and secondary form, depending on the presence or absence of the underlying disease. Primary psoas abscess is caused by bloodstream-mediated infection from a distant site by bacteremia or sepsis. Secondary psoas abscess is usually related to direct spreading or expansion from the adjacent organs and often caused by inflammation and neoplasm of the intrapelvic organs such as inflammatory bowel diseases [23]. Risk factors of psoas muscle abscess include diabetes mellitus, intravenous drug use, immunosuppressive drugs, and human-immunodeficiency-virus infection [29]. Diagnosis often requires imaging such as computed tomography and magnetic resonance imaging and occasionally positron emission tomography. As for the causative bacteria, Staphylococcus aureus is the major pathogen detected in those reported cases. Notably, increasing number of cases is now caused by methicillinresistant Staphylococcus aureus. Treatment involves intravenous administration of sensitive antibiotic agents for at least several weeks and interventions such as needle aspiration or open surgical drainage and debridement of the affected muscles [21]. A previous report showed that the prognosis of secondary psoas abscess is worse than primary psoas abscess, and unless open surgical drainage is performed, its prognosis is unacceptable [30].

Table 3 summarized the previous 22 reports including our case on psoas muscle abscess in hemodialysis patients $[8-10,19-28]$. The age of the patients ranged from 45 to 78 years. Both male and female patients were affected. Primary infection sites included catheters for vascular access, enterocolitis, and discitis. The causative pathogens included Staphylococcus aureus and enteric bacteria, depending on the primary source of infection. 


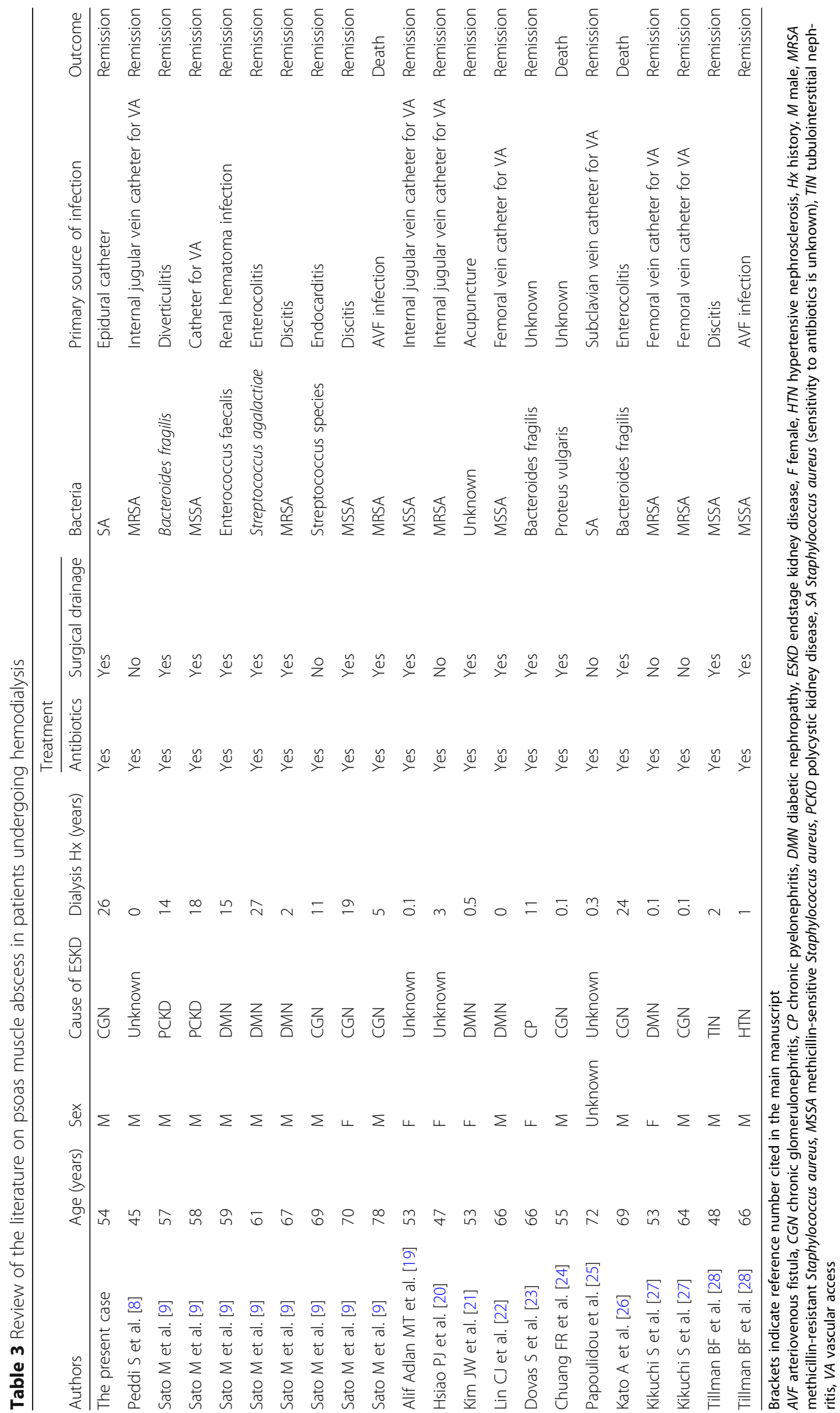


All the catheter-related psoas muscle abscesses were caused by methicillin-sensitive or resistant Staphylococcus aureus. Most cases are treated with combination of intravenous antibiotics treatment and surgical drainage, leading to remission of psoas muscle abscess. As for psoas muscle abscess secondary to catheter-related infection, all previous cases were related to catheter used for vascular access and catheters inserted into the jugular, subclavian, or femoral veins for temporal or permanent use $[7,8,19,20,22,25,27]$. In this regard, our case is the first to report psoas muscle abscess caused by epidural catheter used for continuous epidural anesthesia.

Bacterial infection in the erector spinae muscle is not so common. Staphylococcus aureus occasionally causes abscess in the erector spinae muscle, named tropical myositis, which is restricted in the tropical regions $[31,32]$. However, this disease entity is somewhat different from erector spinae muscle abscess caused by bacteremia. To our knowledge, abscess in the erector spinae muscle has not been reported in dialysis population. In the present case, Staphylococcus aureus was the causative pathogen and successfully treated by sensitive antibiotics. Our case indicates that abscess in the erector spinae muscle can be the possible cause of back pain in a particular medical condition. As for the route of abscess in the erector spinae muscle, two potential pathways were presumed. One was via bloodstream-mediated dissemination and should be prevented by controlling the primary infection promptly. Another was via the route made by lumbar punctures for continuous epidural anesthesia. Because lumbar puncture through para-median pathway penetrates erector spinae muscles, multiple punctures could cause infection of that muscle. In this regard, it may be said that epidural anesthesia may sometimes cause spinae muscle abscess especially in hemodialysis patients because epidural puncture is relatively difficult to do on dialysis patients and sometimes needs multiple punctures via the para-median pathway.

Abscess in the vastus lateralis muscles caused by non-tuberculous bacteria has rarely been reported both in the general population and hemodialysis patients. Since muscle abscess without trauma is usually caused by contamination of the bacteria into the bloodstream, it is reasonable to think that the site of abscess may be stochastic and there may be no common site of muscle abscess. Or blood supply to muscle may be more diverse depending on the location of the muscle, since the psoas muscle is known to have a huge blood supply compared to other muscles. This may be one of the reasons why cases of psoas muscle abscess have been the most. The mechanisms underlying bacterial lodging to muscle may be more complicated. Hence, further studies are necessary to determine why some muscles are prone to develop abscess and others are not under bacteremia or sepsis.

Drainage at an appropriate timing is critical in the management of deep muscle abscess including psoas muscle abscess. Open surgical drainage and percutaneous drainage are the two common modalities for abscess drainage. However, a previous study showed that open surgical drainage reduced total hospital days compared with needle aspiration, especially when abscess is larger [33]. As shown in Table 3, most of the remission cases experienced drainage as well as administration of sensitive antibiotics. In our case, open surgical drainage was performed at 29 days after psoas muscle abscess was detected by imaging. In our case, delayed surgical drainage might be responsible for the delayed remission of our case. One of the important messages of our case was that open surgical drainage without delay is necessary when abscess is larger and administration of sensitive antibiotics for 5-7 days is ineffective [26]. The importance of open surgical drainage is probably explained by the fact that delivery rate of antibiotic to the abscess site is lower than that of other infection sites [34].

As for the route of infection, primary deep muscle infection including iliopsoas muscle abscess is usually mediated by the bloodstream, namely secondary to bacteremia and/or sepsis $[10,18,28]$. However, judging from the distribution and progression of the multiple abscesses in the left-sided muscles, we cannot deny the possibility that abscess in the left iliopsoas muscle expanded into abscess in the left gluteus muscle and vastus lateralis muscle. As for dialysis population, vascular access is often the primary source of infection, even when vascular access infection looks absent [35]. In our case, considering that the epidural space is not directly connected to the iliopsoas muscle and erector spinae muscles and the muscle abscesses distributed bilaterally in our case, it is plausible to think that dissemination of Staphylococcus aureus in the epidural space via the bloodstream was ascribed to the multiple deep muscle abscesses. These results indicate that early control of bacterial meningitis with appropriate antibiotics is critically important to prevent secondary bacteremia and distant organ infection such as muscle abscess that can lead to life-threatening conditions.

Finally, it would be interesting and educational to discuss the best approach to this case. When purulent discharge from the insertion site of epidural catheter is noted, the catheter should be removed and purulent discharge and blood should be cultured to detect the causative organism. Repeat blood cultures until the causative pathogen is detected. Until the causative bacteria are identified, empiric therapy should be started with broadspectrum antibiotics covering Staphylococcus aureus and Gram-positive bacteria, since these bacteria are the two 
major pathogens for epidural catheter-related infection. According to the guideline for bacterial meningitis in Japan, carbapenem and third-generation cephalosporins such as ceftriaxone are recommended because these drugs show a good penetration to the central nervous system [36, 37]. In recent years, the incidence of psoas abscess caused by methicillin-resistant Staphylococcus aureus is increasing. Hence, vancomycin should be included. Once the causative bacteria is detected and its susceptibility to antibiotics is determined, unneeded antibiotics should be discontinued and sensitive antibiotics should be continued for at least 4-6 weeks. If patients complicate bacteremia/septicemia/sepsis and distant organ infection is suspected as in the present case; imaging such as computed tomography and magnetic resonance imaging should be immediately performed to identify the location of the distant organ infection, then consider if there is a need for surgical intervention. Echocardiography should also be performed since dialysis population often are at increased risk for infective endocarditis. When the presence of intravascular infection or bloodstream-associated infection is suspected, continue treatment with sensitive antibiotics for weeks after blood culture becomes negative.

\section{Conclusions}

We presented here the case of a male patient receiving maintenance hemodialysis, whose bacterial meningitis and multiple abscess in the deep muscles were related to the indwelling epidural catheter and were successfully treated with antibiotics treatment and open surgical drainage. Our case confirms that treatment with sensitive antibiotics and open surgical drainage at an appropriate time is necessary to secondary deep muscle abscess in hemodialysis patients. Furthermore, our case suggests that epidural anesthesia may not be a good therapeutic option for the control of herpes zosterrelated pain because patients with active herpes zoster is immunologically more compromised during and shortly after reactivation of VZV than when they are not affected by herpes zoster, especially in hemodialysis patients, who are already immunologically more compromised than the general population.

\section{Availability of data and materials}

The data and materials were all included in the manuscript. However, if needed, authors will submit the other data and imaging of the patient.

\section{Authors' contributions}

SY mainly drafted the manuscript. SY, NT, and NN performed acquisition of data and interpretation of data. KM, TM, and TK revised the manuscript. KT is responsible for the final version of the manuscript. All authors read and approved the final manuscript.

\section{Ethics approval and consent to participate}

A written informed consent was given by the patient to participate in this case report study.
Consent for publication

A written informed consent was given by the patient to publish this case report.

Competing interests

The authors declare that they have no competing interests.

\section{Publisher's Note}

Springer Nature remains neutral with regard to jurisdictional claims in published maps and institutional affiliations.

\section{Author details}

1Department of Medicine and Clinical Science, Graduate School of Medical Sciences, Kyushu University, 3-1-1 Maidashi, Higashi-ku, Fukuoka 812-8582, Japan. ${ }^{2}$ Division of Nephrology, Maeda Hospital, 2742-1 Tachibana-cho, Imari, Saga 848-0027, Japan. ${ }^{3}$ Department of Integrated Therapy for Chronic Kidney Disease, Graduate School of Medical Sciences, Kyushu University, 3-1-1

Maidashi, Higashi-ku, Fukuoka 812-8582, Japan.

Received: 8 August 2017 Accepted: 25 April 2018

Published online: 23 May 2018

References

1. Renal Data System US. USRDS 2010 annual data report: atlas of chronic kidney disease and end-stage renal disease in the United States. Bethesda, MD: National Institute of Diabetes and Digestive and Kidney Diseases, National Institutes of Health; 2010.

2. Nakai $\mathrm{S}$, Iseki K, Itami $\mathrm{N}$, et al. Overview of regular dialysis treatment in Japan (as of 31 December 2009). Ther Apher Dial. 2012;16:11-53.

3. Chonchol M. Neutrophil dysfunction and infection risk in end-stage renal disease. Semin Dial. 2006;19:291-6.

4. Eleftheriadis T, Antoniadi G, Liakopoulos V, Kartsios C, Stefanidis I. Disturbances of acquired immunity in hemodialysis patients. Semin Dial. 2007:20:440-51.

5. Dalrymple LS, Mu Y, Nguyen DV, et al. Risk factors for infection-related hospitalization in in-center hemodialysis. Clin J Am Soc Nephrol. 2015;10: 2170-80.

6. Kanaa M, Wright MJ, Akbani H, et al. Cathasept line lock and microbial colonization of tunneled hemodialysis catheters: a multicenter randomized controlled trial. Am J Kidney Dis. 2015;66:1015-23.

7. Johns TS, Mokrzycki MH. Optimal approach for the diagnosis of hemodialysis catheter-related bacteremia. Clin J Am Soc Nephrol 2016 in press.

8. Peddi S, Ram R, Boreddy VD, et al. Multiple metastatic infections in a hemodialysis patient with untunneled internal jugular catheter. Hemodial Int. 2014;18:192-4

9. Sato M, Iwasa Y, Otsubo S, et al. Psoas abscess in hemodialysis patients. Int Urol Nephrol. 2010:42:1113-6.

10. Okada A, Hangai M, Oda T. Bacteremia with an iliopsoas abscess and osteomyelitis of the femoral head caused by enterococcus avium in a patient with end-stage kidney disease. Intern Med. 2015;54:669-74.

11. Steiner I, Kennedy PG, Pachner AR. The neurotropic herpes viruses: herpes simplex and varicella-zoster. Lancet Neurol. 2007;6:1015-28.

12. Kuo CC, Lee CT, Lee IM, Ho SC, Yang CY. Risk of herpes zoster in patients treated with long-term hemodialysis: a matched cohort study. Am J Kidney Dis. 2012;59:428-33.

13. Sampathkumar P, Drage LA, Martin DP. Herpes zoster (shingles) and postherpetic neuralgia. Mayo Clin Proc. 2009;84:274-80.

14. Cook TM, Counsell D, Wildsmith JA: Royal College of Anaesthetists third National Audit Project. Major complications of central neuraxial block: report on the third National Audit Project of the Royal College of Anaesthetists. Br J Anaesth 2009;102:179-190.

15. Reynolds F. Neurological infections after neuraxial anesthesia. Anesthesiol Clin. 2008;26:23-52.

16. Gershon AA, Breuer J, Cohen Jl, et al. Varicella zoster virus infection. Nat Rev Dis Primers. 2015:1:15016.

17. Gershon AA, Gershon MD, Breuer J, et al. Advances in the understanding of the pathogenesis and epidemiology of herpes zoster. J Clin Virol. 2010; 48(Suppl 1):S2-7.

18. Shields D, Robinson P, Crowley TP. lliopsoas abscess - a review and update on the literature. Int J Surg. 2012;10:466-9. 
19. Alif Adlan MT, Wan Mohd Rasis WA, Mohd Ramadhan MD. Fever with intradialytic pelvic pain: a case of iliopsoas abscess complicated with methicillin-sensitive staphylococcus aureus bacteraemia in an end stage renal failure patient. Med J Malaysia. 2016;71:72-3.

20. Hsiao PJ, Tsai MH, Leu JG, Fang YW. Iliopsoas abscess as a complication of tunneled jugular vein catheterization in a hemodialysis patient. Hemodial Int. 2015;19:330-2.

21. Kim JW, Kim YS. Psoas abscess formation after acupuncture in a hemodialysis patient. Hemodial Int. 2010;14:343-4.

22. Lin CJ, Lin HC, Wu CJ, Chen HH, Chen YC. Psoas muscle abscess as a complication of femoral vein catheterization in a hemodialysis patient South Med J. 2008;101:566-7.

23. Dovas $\mathrm{S}$, Liakopoulos $\mathrm{V}$, Simopoulou T, et al. Psoas abscess in a dialysis patient with dialysis-related amyloidosis. Int Urol Nephrol. 2008;40:543-6.

24. Chuang FR, Lee CH, Chen JB, et al. Extra-renal abscess in chronic hemodialysis patients. Ren Fail. 2002;24:511-21.

25. Papoulidou F, Giannopoulos G, Pliakogiannis T, et al. Septic myositis in a chronic dialysis patient. Clin Nephrol. 2001;56:175-7.

26. Kato A, Takahashi T, Watanabe T, Furuhashi M, Maruyama Y, Hishida A. Psoas abscess with osteomyelitis in a patient undergoing long-term hemodialysis. Am J Nephrol. 2001;21:410-2.

27. Kikuchi S, Muro K, Yoh K, et al. Two cases of psoas abscess with discitis by methicillin-resistant Staphylococcus aureus as a complication of femoralvein catheterization for haemodialysis. Nephrol Dial Transplant. 1999;14: 1279-81.

28. Tillman BF, Gibson RL, Stone WJ. Psoas abscess in chronic dialysis patients. J Urol. 1987;137:489-90.

29. Mallick IH, Thoufeeq MH, Rajendran TP. lliopsoas abscesses. Postgrad Med J. 2004;80:459-62.

30. Ricci MA, Rose FB, Meyer KK. Pyogenic psoas abscess: worldwide variations in etiology. World J Surg. 1986;10:834-43.

31. Marshman LA, Bhatia CK, Krishna M, Friesem T. Primary erector spinae pyomyositis causing an epidural abscess: case report and literature review. Spine J. 2008;8:548-51.

32. Zheng YC, Chen CC, Wei KC, et al. Tropical pyomyositis of erector spinae complicated with spinal epidural abscess. Clin Neurol Neurosurg. 2015;128:84-9.

33. Santaella RO, Fishman EK, Lipsett PA. Primary vs secondary iliopsoas abscess. Presentation, microbiology, and treatment. Arch Surg. 1995;130:1309-13.

34. Obata A, Kaneto H, Kamei S, et al. Case of iliopsoas abscess that was markedly recovered after percutaneous and surgical drainage in a patient with poorly controlled type 2 diabetes. J Diabetes Investig. 2016;7:440-1.

35. Camins BC. Prevention and treatment of hemodialysis-related bloodstream infections. Semin Dial. 2013;26:476-81.

36. Ishikawa H, Kamei S. Revised Japanese guidelines for the clinical management of bacterial meningitis. Rinsho Shinkeigaku. 2014;54:1021-3.

37. Japanese Society of Chemotherapy Committee on guidelines for treatment of anaerobic infections; Japanese Association for Anaerobic Infection Research. Chapter 3-5. Appendix: pharmacokinetics of antimicrobial agents indicated for anaerobic infections. J Infect Chemother. 2011;17(Suppl 1):176-9.

\section{Ready to submit your research? Choose BMC and benefit from:}

- fast, convenient online submission

- thorough peer review by experienced researchers in your field

- rapid publication on acceptance

- support for research data, including large and complex data types

- gold Open Access which fosters wider collaboration and increased citations

- maximum visibility for your research: over $100 \mathrm{M}$ website views per year

At BMC, research is always in progress.

Learn more biomedcentral.com/submissions 